Ann. rheum. Dis. (1979), 38, Supplement p. 96

\title{
Workshop III. Management of Reiter's syndrome
}

\section{Management of Reiter's syndrome}

\author{
ANDREI CALIN \\ From the Stanford University Medical Centre, California, USA
}

Reiter's syndrome (RS) may be defined as shown in the Table. The various manifestations may provide a triad, tetrad, pentad, or hexad of features. Depending on the severity of the disease, the time at which the patient is seen, the obsession of the physician, and other variables, few or many characteristics may be found. For example, the patient may present with only urethritis and Achilles tendinitis or may have all the features shown in the Table ${ }^{53}$ as well as fever, anaemia, cardiac disease, and other manifestations ${ }^{52}$.

There is no cure for RS. Few rheumatic syndromes are more frustrating to treat. False expectations of the patient (and sometimes physician) often result in doctor 'shopping'. The plethora of new non-steroidal anti-inflammatory drugs has not improved therapy*. Explanation is a major part of management. The disease is often bewildering and disturbing for the patient and family. Feelings of guilt and anxiety about sexual misconduct should be allayed. Although anecdotal evidence suggests that certain patients with post-venereal RS are more likely to relapse after a sexual indiscretion, many develop an exacerbation without obvious cause.

It may be useful to provide the patient with an 'allergic' analogy - that some people have a tendency to develop asthma or hayfever on exposure to known or unknown allergens while others have a genetically determined susceptibility to develop RS after contact with an unrecognised (or recognised) precipitating event. This approach provides the patient with some insight into the recurrent nature of the condition and aids discussion about prognosis.

Although there are no control data, there is some suggestion that the use of a condom protects the patient from a post-venereal exacerbation. Apart

*In our experience arthritis or enthesiopathy dan in a sizeable proportion of patients continue unabated despite antiinflammatory treatment to tolerance (for example, phenylbutazone $800 \mathrm{mg} /$ day)-Editor.
Table Definition of Reiter's syndrome

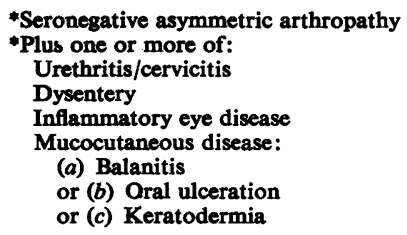

*Seronegative asymmetric arthropathy

-Plus one or more of:

Urethritis/cervicitis

Dysentery

Inflammatory eye disease

Mucocutaneous disease:

(a) Balanitis

or (b) Oral ulceration

or (c) Keratodermia

*Exclusions: primary ankylosing spondylitis, psoriatic arthropathy, other rheumatic disease.

from this manoeuvre and the recommended avoidance of multiple sexual partners there is no way to avoid recurrent attacks or chronicity.

\section{Drug treatment}

Symptomatic management of the acute episode includes the use of analgesics, non-steroidal antiinflammatory agents, and modalities of physical therapy. Antibiotic treatment is a matter of controversy. One uncontrolled study recommended the use of lincomycin, ${ }^{232}$ but others ${ }^{58,175}$ showed that antibiotics did not influence the development of RS in patients with non-specific urethritis.

Joint and soft tissue manifestations seem to respond better to indomethacin, phenylbutazone, and other newer non-steroidal anti-inflammatory drugs than to aspirin, but there have been no controlled studies. Indomethacin $25 \mathrm{mg}$ to $50 \mathrm{mg}$ three times a day may decrease the inflammatory process. If it is either not tolerated or not efficacious phenylbutazone $100 \mathrm{mg}$ three or four times daily may be given. Whether long-term therapy decreases the chance to relapse or chronicity is unknown.

Patients with severe inflammation of the uveal tract may require corticosteroid eye drops or subconjunctival injections. But, apart from an 
anecdotal suggestion that systemic corticosteroids are useful, ${ }^{240}$ they seem to have no role in management. Indeed, a failure to respond to corticosteroids helps to differentiate an enthesopathic disorder from a synovitis. Intralesional therapy with methyl prednisolone (40 to $80 \mathrm{mg}$ ) may be helpful for persistent monoarthropathy, plantar fasciitis, or Achilles tendinitis.

Patients with relentlessly progressing disease require more aggressive treatment. Despite the risks of cytotoxic drugs experience with psoriatic arthropathy ${ }^{23}$ suggests that they may be useful. We usually give azathioprine 1.5 to $2.5 \mathrm{mg} / \mathrm{kg}$ body weight daily. Uncontrolled data suggest that methotrexate may also be suitable. ${ }^{105,}{ }^{233}$ Treatment should be continued until there is improvement, usually within two to six months, and then the dosage tapered off, as able. Support for the use of stilboestrol ${ }^{192}$ has not been critically analysed.

$\mathbf{R S}$ is a major chronic rheumatic disease. Treatment is empirical and inadequate. Efforts should be made towards improving diagnosis, elucidating aetiological factors, and, I hope, providing a cure.
Meanwhile various treatments should be subjected to controlled study.

\section{General discussion}

PROF. B. AMOR: In a chronic form of RS we often use gold salts with good results after three to four months of inadequate response to non-steroid anti-inflammatory treatment. We think this approach preferable to methotrexate or any other cytotoxic drug.

DR. CALIN: Indeed, we are about to start a controlled co-operative study comparing penicillamine and gold in treating seronegative spondarthritides.

PROF. A. S. RUSSELL: In preparation for this symposium I looked really hard for cases of RS that were refractory to drug treatment. Unlike Dr. Calin, I was unable to find them. Our experience corresponds to that of Dr. Ford and Dr. Arnett and I do not think chronic RS is common. We have had a few chronic progressive cases of RS that have presented with spondylitis and skin disease. We have used methotrexate in only two or three of them, with relative success in clearing the skin disease. 